\title{
The Best Piecewise Linearization of Nonlinear Functions
}

\author{
Mohammad Mehdi Mazarei*, Ali Asghar Behroozpoor, Ali Vahidian Kamyad \\ Department of Applied Mathematics, School of Mathematical Sciences, Ferdowsi University of Mashhad, \\ International Campus, Mashhad, Iran \\ Email: ${ }^{*}$ mm.mazarei@pgstp.ir
}

Received 11 August 2014; revised 12 September 2014; accepted 8 October 2014

Copyright @ 2014 by authors and Scientific Research Publishing Inc.

This work is licensed under the Creative Commons Attribution International License (CC BY).

http://creativecommons.org/licenses/by/4.0/

c) (i) Open Access

\begin{abstract}
In this paper, we propose a method for finding the best piecewise linearization of nonlinear functions. For this aim, we try to obtain the best approximation of a nonlinear function as a piecewise linear function. Our method is based on an optimization problem. The optimal solution of this optimization problem is the best piecewise linear approximation of nonlinear function. Finally, we examine our method to some examples.
\end{abstract}

\section{Keywords}

\section{Nonlinear Systems, Piecewise Linearization, Optimization Problem, Linear Programming}

\section{Introduction}

The linearization of nonlinear systems is an efficient tool for finding approximate solutions and treatment analysis of these systems, especially in application [1]-[3]. Some researchers have used some methods based on the optimization problem [4]. But in many applications for nonlinear and nonsmooth functions, we are faced to some problems. In fact, piecewise linearization is a more efficient tool for finding approximate solutions. Some researchers have used piecewise linearization in applications [5] [6]. Also, some researchers have used piecewise linearization to solve ODEs and PDEs [7].

First, we consider a nonlinear function. Let $F: A \subseteq R^{n} \rightarrow R$ be a nonlinear function. We suppose that $x=\left(x_{1}, x_{2}, \cdots, x_{n}\right)$ varies in a subset of $R^{n}$ as $A$ and this subset is compact. Our aim is to approximate the nonlinear function $F$ by a piecewise linear function as follows:

$$
f(x)=\sum_{i=1}^{N}\left(a_{i 0}+a_{i 1} x_{1}+a_{i 2} x_{2}+\cdots+a_{i n} x_{n}\right) \chi_{A_{i}}(x), \quad a_{i j} \in R ; \quad i=1,2, \cdots, N
$$

*Corresponding author. 
where $A_{i}$ is $i$ th subset in partitioning of $A$ as $P_{N}=\left\{A_{1}, A_{2}, \cdots, A_{N}\right\}$. As we know, this partitioning has bellow properties:

1) $\forall i, j=1,2, \cdots, N ; \quad A_{i} \cap A_{j}=\varnothing, A_{i} \in R^{n}$

2) $A=\bigcup_{i=1}^{N} A_{i}$

Also, $\chi_{A_{i}}(x)$ is a characteristic function on $\left\{A_{1}, A_{2}, \cdots, A_{N}\right\}$ such that:

$$
\chi_{A_{i}}(x)= \begin{cases}1, & x \in A_{i} \\ 0, & x \notin A_{i}\end{cases}
$$

Now, let $L_{1}(A)=\left\{f: A \rightarrow R\left|\int_{A}\right| f \mid \mathrm{d} x<\infty\right\}$. As we know $L_{1}(A)$ is a Hilbert space of $A$ with the following inner product:

$$
\langle f, g\rangle=\int_{A} f(x) g(x) \mathrm{d} x, \quad f, g \in L(A)
$$

and

$$
\|f\|_{1}=\int_{A}|f| \mathrm{d} x
$$

Definition 1. We define $S_{N}(A) \quad(N \in \mathbb{N})$ be the set of all $f \in L_{1}(A)$ of the form (1).

Definition 2. If $F: R^{n} \rightarrow R$ is a nonlinear function and $f \in S_{N}(A)$, we define $\|F-f\|_{L_{1}}$ as follows:

$$
\|F-f\|_{L_{1}}=\int_{A}|F-f| \mathrm{d} x
$$

Theorem. The subset $S_{N}(A)$ is dens on $L_{1}(A)$.

Proof. Suppose that $F$ be a nonlinear function that $F: A \subseteq R^{n} \rightarrow R$.

$$
\forall \varepsilon>0 \exists N(\varepsilon) \in \mathbb{N}, \quad \exists f(\cdot) \in S_{N}(A) \ni\|F-f\|_{L_{1}}<\varepsilon
$$

Definition 3. We call $f^{*} \in S_{N}(A)$ the best piecewise linear approximation of $F$ if for any $f \in S_{N}(A)$ we have

$$
\left\|F-f^{*}\right\|_{L_{1}} \leq\|F-f\|_{L_{1}} .
$$

In fact, by above definition $f^{*}$ is optimal solution of the following optimization problem:

$$
\begin{gathered}
\operatorname{Min}\|F-f\|_{L_{1}} \\
f \in S_{N}(A)
\end{gathered}
$$

Obviously, because $0 \leq\|F-f\|_{L_{1}}$, the optimization problem (6) has optimal solution.

\section{Approach}

At first, we consider a nonlinear function $F: R \rightarrow R$. Secondly, we explain this approach for a nonlinear function $F: R^{2} \rightarrow R$. Then, we explain this approach for a nonlinear function $F: R^{n} \rightarrow R$.

1) Let to consider the bellow optimization problem

$$
\begin{gathered}
\operatorname{Min}\|F-f\|_{L_{1}} \\
f \in S_{N}(A)
\end{gathered}
$$

where, $F: A \subseteq R \rightarrow R$ is a nonlinear function and $A=[a, b]$. As we know $[a, b]$ can be replaced by $[0,1]$.

Now, we decompose interval $[0,1]$ to $N$ subintervals $\left[\frac{i-1}{N}, \frac{i}{N}\right] ; i=1,2, \cdots, N$ (See Figure 1).

Since, $f \in S_{N}(A)$, we have 


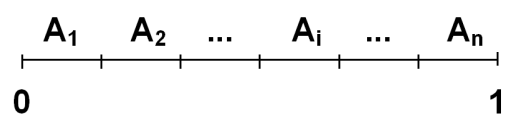

Figure 1. Partitioning $A$ to subintervals $A_{i}$.

$$
\operatorname{Min} \int_{0}^{1}\left|F(x)-\sum_{i=1}^{N}\left(a_{i}+b_{i} x\right) \chi_{\left[\frac{i-1}{N}, \frac{i}{N}\right]}(x)\right| \mathrm{d} x
$$

Our objective function is a functional. Now, we reduce this functional to a summation as follows:

$$
\int_{0}^{1}\left|F(x)-\sum_{i=1}^{N}\left(a_{i}+b_{i} x\right) \chi_{\left[\frac{i-1}{N}, \frac{i}{N}\right]}(x)\right| \mathrm{d} x \simeq \frac{1}{N} \sum_{i=1}^{N}\left|F\left(x_{i}\right)-\left(a_{i}+b_{i} x_{i}\right)\right|
$$

So, the optimization problem (8) is as follows:

$$
\begin{aligned}
& \operatorname{Min} \sum_{i=1}^{N}\left|r_{i}-s_{i}\right| \\
& \quad s . t \\
& r_{i}-s_{i}=F\left(x_{i}\right)-\left(a_{i}+b_{i} x_{i}\right) \\
& 0 \leq r_{i}, s_{i} ; \quad i=1,2, \cdots, N .
\end{aligned}
$$

But, the optimization problem (9) is a nonlinear programming problem. We reduce this problem to a linear programming problem by relation $\left|r_{i}-s_{i}\right|=r_{i}+s_{i}$ such that $r_{i} \cdot s_{i}=0$. So, our optimization problem will be as follows:

$$
\begin{aligned}
& \operatorname{Min} \frac{1}{N} \sum_{i=1}^{N} r_{i}+s_{i} \\
& \quad \text { s.t } \\
& r_{i}-s_{i}=F\left(x_{i}\right)-\left(a_{i}+b_{i} x_{i}\right) \\
& r_{i} \cdot s_{i}=0 \\
& 0 \leq r_{i}, s_{i} ; \quad I=1,2, \cdots, N .
\end{aligned}
$$

2) Second, we consider a nonlinear function $F: A \subseteq R^{2} \rightarrow R$. So, we have the optimization problem as follows:

$$
\operatorname{Min} \int_{A}\left|F(x)-\sum_{i=1}^{N}\left(a_{i}+b_{i} x+c_{i} y\right) \chi_{A_{i}}(x)\right| \mathrm{d} x
$$

where $A_{i}$ is the ith partition in partitioning of $A=[a, b] \times[c, d]$ and $\mathrm{d} x=\mathrm{d} x \mathrm{~d} y$. Also we can replace $A$ by $[0,1] \times[0,1]$. As, we explained in 1 ) the optimization problem (11) will be reduced to a linear programming problem as follows:

$$
\begin{aligned}
& \operatorname{Min} \sum_{i=1}^{N} r_{i}+s_{i} \\
& \quad \text { s.t } \\
& r_{i}-s_{i}=F\left(x_{i}, y_{i}\right)-\left(a_{i}+b_{i} x_{i}+c_{i} y_{i}\right) \\
& r_{i} \cdot s_{i}=0 \\
& 0 \leq r_{i}, s_{i} \\
& i=1,2, \cdots, N \\
& N=m \times n .
\end{aligned}
$$

where $m$ and $n$ are numbers of subintervals on axises $x$ and $y$, respectively (See Figure 2).

3) Third, we consider a nonlinear function $F: A \subseteq R^{n} \rightarrow R$. So, we have the optimization problem as fol- 


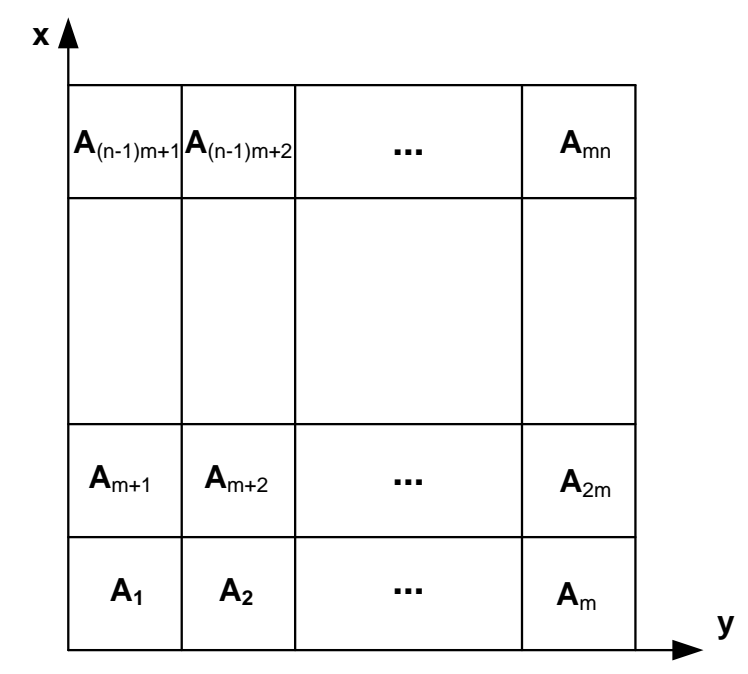

Figure 2. Partitioning $A$ to subintervals $A_{i}$.

lows:

$$
\operatorname{Min} \int_{A}\left|F\left(x_{1}, x_{2}, \cdots, x_{n}\right)-\sum_{i=1}^{N}\left(a_{i 0}+a_{i 1} x_{1}+a_{i 2} x_{2}+\cdots+a_{i n} x_{n}\right) \chi_{A_{i}}(x)\right| \mathrm{d} x
$$

As, we explained in sections 1) and 2) this optimization problem will be reduced to a linear programming problem as follows:

$$
\begin{aligned}
& \operatorname{Min} \sum_{i=1}^{N} r_{i}+s_{i} \\
& \quad s . t \\
& r_{i}-s_{i}=F\left(x_{1}, x_{2}, \cdots, x_{n}\right)-\left(a_{i 0}+a_{i 1} x_{1}+a_{i 2} x_{2}+\cdots+a_{i n} x_{n}\right) \\
& r_{i} \cdot s_{i}=0 \\
& 0 \leq r_{i}, s_{i} ; \quad i=1,2, \cdots, N \\
& N=m_{1} \times m_{2} \times \cdots \times m_{n} .
\end{aligned}
$$

where $m_{1}, m_{2}, \cdots, m_{n}$ are numbers of subintervals on axises $x_{1}, x_{2}, \cdots, x_{n}$ respectively.

\section{Examples}

In this section, we show efficiency of our approach by several examples. Also, we define the root mean squared error by follow relation:

$$
\text { RMSE }=\sqrt{\frac{\sum_{i=1}^{N} \mathrm{e}_{i}^{2}}{N}}
$$

Example 1. We consider nonlinear nonsmooth function $F(x)=|x-.2|$ on interval $[0,1]$.

As we explained in section 1), the linear programming corresponding to this function is as follows:

$$
\begin{aligned}
& \operatorname{Min} \frac{1}{N} \sum_{i=1}^{N} r_{i}+s_{i} \\
& \quad s . t \\
& r_{i}-s_{i}=\left|x_{i}-.2\right|-\left(a_{i}+b_{i} x_{i}\right) \\
& r_{i} \cdot s_{i}=0 \\
& 0 \leq r_{i}, s_{i} ; \quad I=1,2, \cdots, N .
\end{aligned}
$$


The optimal solution of linear programming problem (16) is the best piecewise linearization of the function $F(x)=|x-.2|$ on $[0,1]$. We let $N=50$ and $N=100$, respectively (See Figure 3, Figure 4). In this example, we have $\mathrm{RMSE}=7.81 \times 10^{-14}$ for $N=100$. As we can see the approximate piecewise linearization of this function is high accurate.

Example 2. We consider nonlinear function $F(x)=x^{2}$ on interval [0,1]. We have obtained the piecewise approximation of this nonlinear function using two other methods. These methods are Splines Piecewise Approximation (SPA) and Mixture of Polynomials (MOP). Then we have compared these with our method.

As we explained in section 1), the linear programming corresponding to this function is as follows:

$$
\begin{aligned}
& \operatorname{Min} \frac{1}{N} \sum_{i=1}^{N} r_{i}+s_{i} \\
& \quad s . t \\
& r_{i}-s_{i}=x_{i}^{2}-\left(a_{i}+b_{i} x_{i}\right) \\
& r_{i} \cdot s_{i}=0 \\
& 0 \leq r_{i}, s_{i} ; \quad I=1,2, \cdots, N .
\end{aligned}
$$

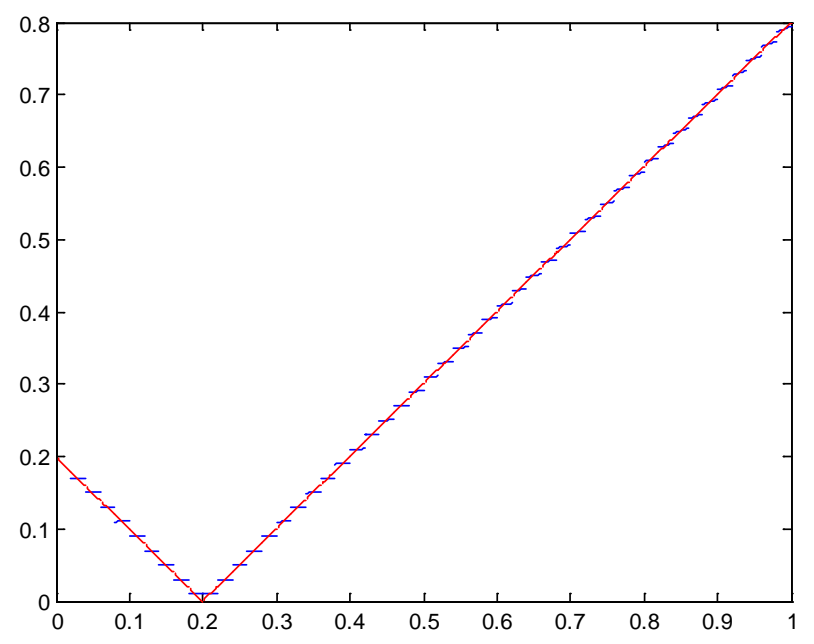

Figure 3. The figure of piecewise function approximation of nonlinear function $F(x)=|x-.2|$ for $N=50$.

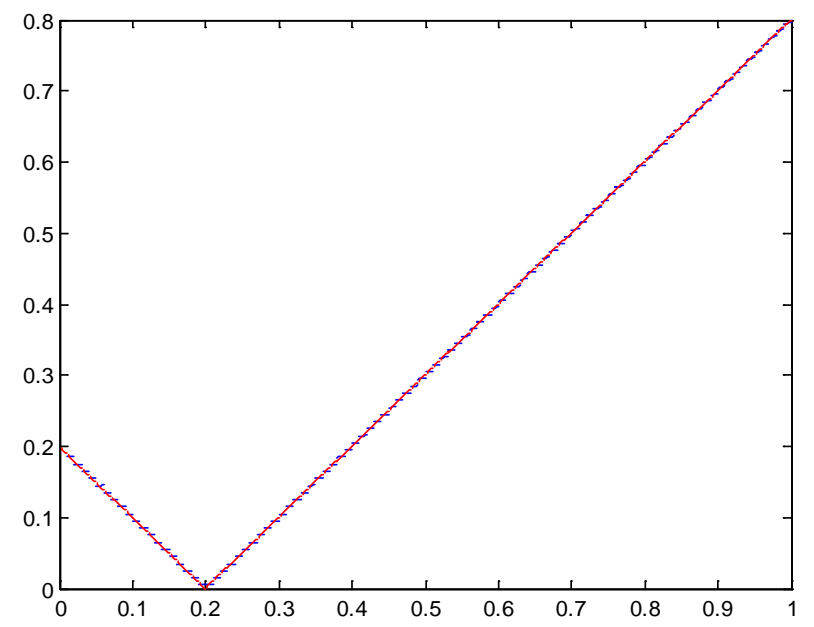

Figure 4. The figure of piecewise function approximation of nonlinear function $F(x)=|x-.2|$ for $N=100$. 
The optimal solution of linear programming problem (17) is the best piecewise linearization of the function $F(x)=x^{2}$ on $[0,1]$. We let $N=100$ (See Figure 5). In this example, we have RMSE $=7.52 \times 10^{-12}$ while RMSE $=2.26 \times 10^{-5}$ for Splines Piecewise Approximation and RMSE $=5.03 \times 10^{-7}$. As we can see the approximate piecewise linearization of this function using our method is more accurate in compared with two other methods.

Example 3. We consider nonlinear non smooth function $F(x, y)=|x-y|$ on $[0,1] \times[0,1]$.

As we explained in Section 2), the linear programming corresponding to this function is as follows:

$$
\begin{aligned}
& \operatorname{Min} \sum_{i=1}^{N} r_{i}+s_{i} \\
& \text { s.t } \\
& r_{i}-s_{i}=\left|x_{i}-y_{i}\right|-\left(a_{i}+b_{i} x_{i}+c_{i} y_{i}\right) \\
& r_{i} \cdot s_{i}=0 \\
& 0 \leq r_{i}, s_{i} \\
& i=1,2, \cdots . N \\
& N=m \times n \text {. }
\end{aligned}
$$

The optimal solution of linear programming problem (18) is the best piecewise linearization of the function $F(x, y)=|x-y|$ on $[0,1]$. We let $m=n=10$ and $m=n=40$ (See Figure 6, Figure 7). In this example,

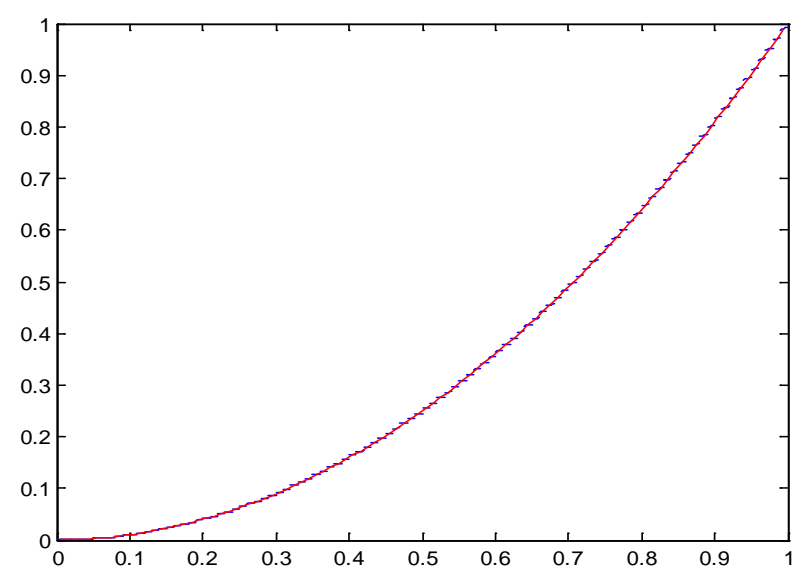

Figure 5. The figure of piecewise function approximation of nonlinear function $F(x)=x^{2}$ for $N=100$.

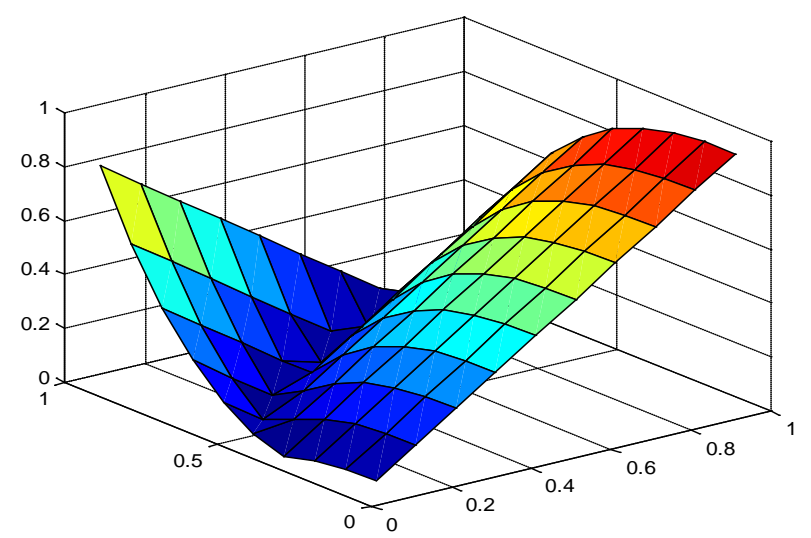

Figure 6. The figure of piecewise function approximation of nonlinear function $F(x, y)=|x-y|$ for $m=n=10$. 


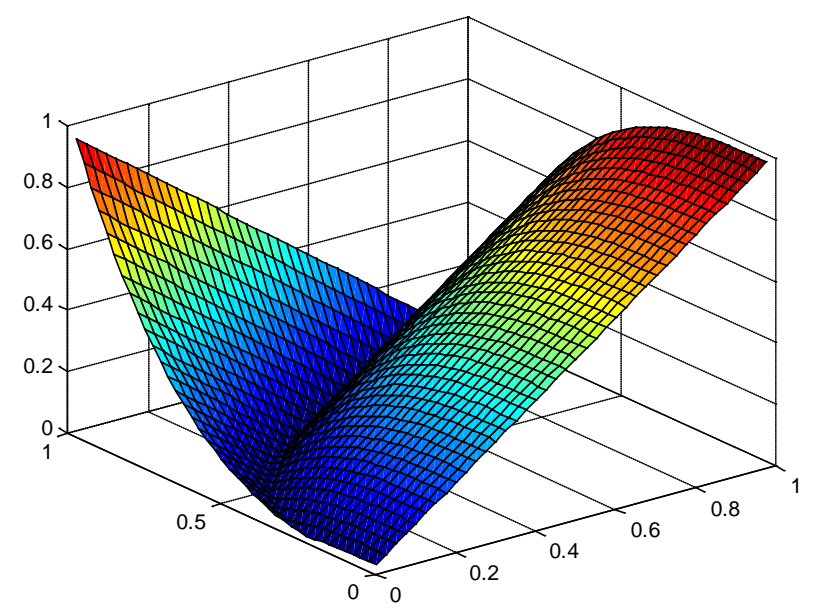

Figure 7. The figure of piecewise function approximation of nonlinear function $F(x, y)=|x-y|$ for $m=n=40$.

we have $\mathrm{RMSE}=1.28 \times 10^{-14}$ and $\mathrm{RMSE}=7.97 \times 10^{-13}$, respectively. As we can see the approximate piecewise linearization of this function is high accurate.

\section{Conclusion}

Our method for piecewise linearization of nonlinear functions is extensible to $R=(-\infty,+\infty)$ by the function $:(-\infty,+\infty) \rightarrow[0,1]$. As we can see, this approximation is high accurate in comparison of other methods and this method is very simple for achieving this optimal solution. Also, this piecewise linearization form of nonlinear functions is useful for many applications, especially for nonlinear nonsmooth optimization, nonlinear differential equations, fuzzy ODE and PDE differential equations and so on.

\section{References}

[1] Aranda-Bricaire, E., Kotta, U. and Moog, C. (1996) Linearization of Discrete-Time Systems. SIAM Journal on Control and Optimization, 34, 1999-2023. http://dx.doi.org/10.1137/S0363012994267315

[2] Jouan, P. (2003) Immersion of Nonlinear Systems into Linear Systems Modulo Output Injection. SIAM Journal on Control and Optimization, 41, 1756-1778. http://dx.doi.org/10.1137/S0363012901391706

[3] Sladeček, L. (2003) Exact Linearization of Stochastic Dynamical Systems by State Space Coordinate Transformation and Feedback Ig-Linearization. Applied Mathematics E-Notes, 3, 99-106.

[4] Vahidian Kamyad, A., Hashemi Mehne, H. and Hashemi Borzabadi, A. (2005) The Best Linear Approximation for Nonlinear Systems. Applied Mathematics and Computation, 167, 1041-1061. http://dx.doi.org/10.1016/j.amc.2004.08.002

[5] Herdem, S. and Koksal, M. (2002) A Fast Algorithm to Compute Steady-State Solution of Nonlinear Circuits by Piecewise Linearization. Computers and Electrical Engineering, 28, 91-101.

[6] Gunnerud, V., Foss, B.A., Mckinnon, K.I.M. and Nygreen, B. (2012) Oil Production Optimization Solved by Piecewise Linearization in a Branch and Price Framework. Computers and Operations Research, 39, 2469-2477. http://dx.doi.org/10.1016/j.cor.2011.12.013

[7] Ramos, J.I. and Garcia-Lopez, C.M. (1997) Nonstandard Finite Difference Equations for ODEs and 1-D PDEs Based on Piecewise Linearization. Applied Mathematics and Computations, 86, 11-36. 
Scientific Research Publishing (SCIRP) is one of the largest Open Access journal publishers. It is currently publishing more than 200 open access, online, peer-reviewed journals covering a wide range of academic disciplines. SCIRP serves the worldwide academic communities and contributes to the progress and application of science with its publication.

Other selected journals from SCIRP are listed as below. Submit your manuscript to us via either submit@scirp.org or Online Submission Portal.
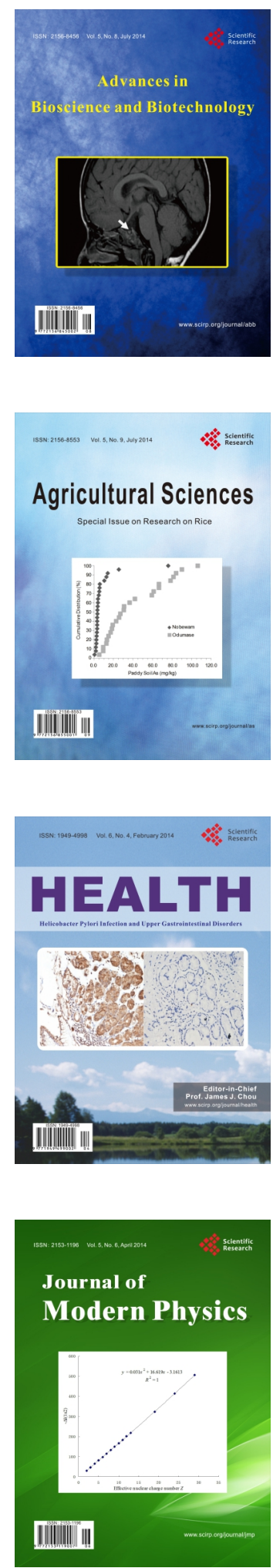
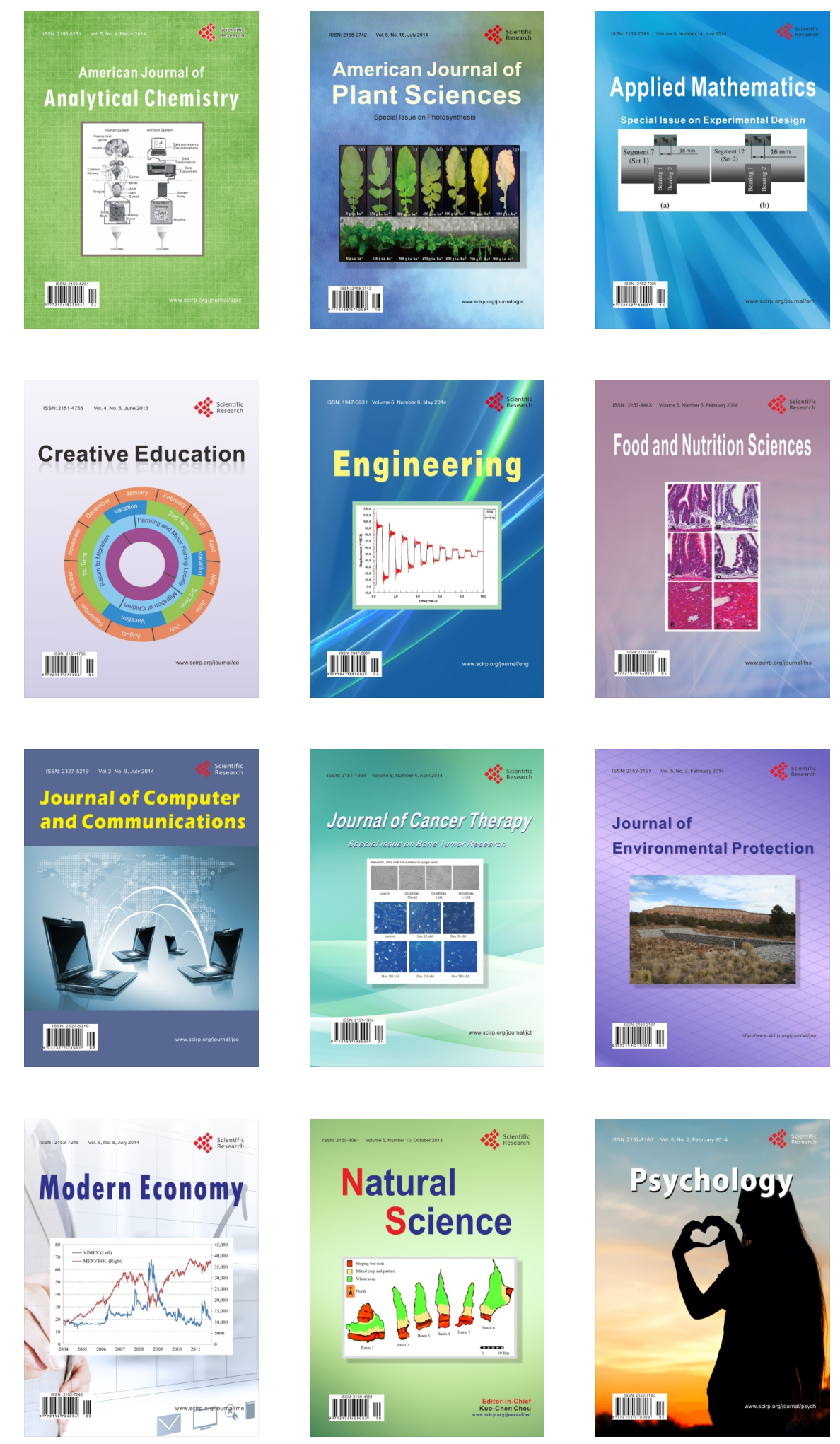